Pacific Journal of Mathematics

NONLINEAR SMOOTH REPRESENTATIONS OF COMPACT LIE 


\title{
NONLINEAR SMOOTH REPRESENTATIONS OF COMPACT LIE GROUPS
}

\author{
M. ROTHENBERg AND J. Sondow
}

We study semi-free (=free off the fixed-point set) smooth actions of a compact Lie group $G$ on disks and spheres with fixed-point set a disk or sphere, respectively. In dimensions $\geqq 6$ and codimension $\neq 2$ we obtain a complete classification for such actions on disks and a partial classification for spheres, together with partial results in dimension 5 or codimension 2. We show that semi-free smooth actions of $G$ on the $n$-disk $D^{n}, n \geqq 6+\operatorname{dim} G$, with fixed-point set an $(n-k)$-disk, $k \neq 2$, are classified by two invariants:

(1) a free orthogonal action of $G$ on the $(k-1)$-sphere $S^{k-1}$ (the representation at the fixed points) and

(2) an element of the Whitehead group $\mathrm{Wh}\left(\pi_{\circ}(G)\right)$.

In fact $(\S 4$, Theorem $\mathrm{A})$, there is a bijection $\tau$ from the set $\mathscr{D}_{\rho}^{n, k}$ of such actions, with a given representation $\rho: G \rightarrow O(k)$ at the fixed points, onto $\mathrm{Wh}\left(\pi_{\circ}(G)\right.$ ), and for $n-k \geqq 2, \mathscr{D}_{p}^{n, k}$ is a group under equivariant boundary connected sum and $\tau$ is an isomorphism. The corresponding set $\mathscr{S}_{p}^{n, k}$ of actions on spheres also forms a group (§ 4, Corollary 4).

For $G=Z_{m}=Z / m Z$ we show ( $\$ 4$, Corollary 5) that these actions on $D^{n}$ restrict to distinct actions on $\partial D^{n}=S^{n-1}$ if $n-1$ is odd. For $n=k$ these actions on $S^{n-1}$ are free (since $S^{n-k-1}=S^{-1}=\varnothing$ ) and, in fact, are the same as those constructed by Milnor in [20], where he used Reidemeister torsion to distinguish infinitely many of them. We observe that his later application [21, Corollary 12.13] of the Atiyah-Bott fixed-point formula [1, §7] implies they are all distinct. For $n>k$ we use Whitehead torsion to distinguish them, employing the result of [11] and [3, Prop. 4.14] that $\mathrm{Wh}\left(Z_{m}\right)$ is free abelian. (For analogous applications of Reidemeister torsion versus Whitehead torsion com- pare [19] vs. [37] and [33, 36] vs. [31].)

Thus for $m \neq 1,2,3,4$ or 6 , which according to [11] imslies $\operatorname{rank} \mathrm{Wh}\left(Z_{m}\right)>0$, we obtain $(\S 4$, Corollary 6) infinitely many different semi-free smooth actions of $Z_{m}$ on every sphere of odd dimension greater than four, with fixed-point set a sphere of any even codimension at least four. These actions are not smoothly equivalent to linear actions, although they are topologically linear according to [38] and [9].

Invariant (1) of a semi-free action is equivalent to a representation $\rho: G \rightarrow O(k)$ that is "fixed-point-free", i.e., such that $\rho(g)$ has no eigenvalue equal to +1 for $g \in G, g \neq$ identity. The only $G$ which 
admit such a representation are the finite "fixed-point-free" groups classified by Wolfe [40, Theorems 6.1.11 and 6.3.1] and $S^{1}, S^{3}$ and the normalizer of $S^{1}$ in $S^{3}$ (see Bredon [5, p. 153]). We do not use Wolf's classification in this paper, and the only place we use Bredon's result is in $\S 4$, Corollary 2, which states in part that every semi-free smooth action of a compact Lie group $G$ of positive dimension on $D^{n}$ with fixed-point set an $(n-k)$-disk is smoothly equivalent to a linear action if $n \geqq 6+\operatorname{dim} G$ and $k \neq 2$. This is proved without assuming $k \neq 2$ in [5, VI. 9.1].

The rest of the paper is organized as follows. In $\S 1$ we discuss $(G, \rho)$ equivariant orientations and define $\mathscr{D}_{0}^{n, k}$ and $\mathscr{S}_{\rho}^{n, k}$. In $\S 2$ we define equivariant connected sum operations on $\mathscr{D}_{\rho}^{n, k}$ and $\mathscr{S}_{\rho}^{n, k}$, introduce the group $D S_{\rho}^{n k}$ of equivalence classes of $(G, \rho)$ orientationpreserving diffeomorphisms of $S^{n}$ equivariant with respect to the linear action of $G$ on $S^{n}$ induced by $G \stackrel{\rho}{\rightarrow} O(k) \subset O(n+1)$, and define the set $C \mathscr{S}_{\rho}^{n, k}$ (which is a group for $n>k$ ) of $h$-cobordism classes of elements of $\mathscr{S}_{\rho}^{n, k}$. For $n \geqq k$, and for $n=k-1 \geqq 5$ if $G$ is cyclic, these sets are related by an exact sequence

$$
0 \longrightarrow D S_{\rho}^{n, k} \stackrel{\alpha}{\longrightarrow} \mathscr{S}_{\rho}^{n+1, k} \stackrel{\beta}{\longrightarrow} \mathscr{D}_{\rho}^{n+1, k} \stackrel{\partial}{\longrightarrow} \mathscr{S}_{\rho}^{n, k} \stackrel{\nu}{\longrightarrow} C \mathscr{S}_{\rho}^{n, k} \longrightarrow 0
$$

in $\S 3$. Then $\S 4$ contains our main results, including the fact (Corollary 5) that for $G=Z_{m}, n \geqq 5$ odd, and $n \geqq k+2$, the exact sequence of $\S 3$ breaks into

$$
0 \longrightarrow \mathrm{Wh}\left(Z_{m}\right) \stackrel{\partial \circ \tau^{-1}}{\longrightarrow} \mathscr{S}_{\rho}^{n, k} \stackrel{\nu}{\longrightarrow} C \mathscr{S}_{\rho}^{n, k} \longrightarrow 0
$$

and

$$
0 \longrightarrow D S_{\rho}^{n, k} \stackrel{\alpha}{\longrightarrow} \mathscr{S}_{\rho}^{n+1, k} \longrightarrow 0
$$

where $\tau: \mathscr{D}_{\rho}^{n+1, k} \rightarrow \mathrm{Wh}\left(Z_{m}\right)$ is the isomorphism of Theorem $\mathrm{A}$ in $\S 4$. The proofs of some of the results in $\S \S 2,3,4$ are postponed to $\S 5$ in the hope of facilitating the exposition of the earlier sections. Some of our results on $\mathscr{S}_{a}^{n, k}$, for $n=k$ and $G$ cyclic, have also been obtained by Sebastiani [29].

Finally, a word on the genesis of this paper. Some of the results were announced in [34, 35], where semi-free actions were called relatively free. An early version of the paper was first circulated as a preprint in 1969. It appears as a reference in the following papers (this list is not necessarily meant to be exhaustive): [6], [7], [26, 27, 28], [15] and [25], which contains a generalization of Theorem $\mathrm{A}(1) .[23,24]$ are expository lecture notes on the subject. [30] and [39] are related.

1. Definitions. Let $G$ be a compact Lie group. Let $\rho: G \rightarrow$ 
$G L(k)$ be a linear representation, where $G L(k)$ is the general linear group on $k$ real variables. The pair $(G, \rho)$ will be fixed throughout the discussion. We say that $\rho$ and $\rho^{\prime}: G \rightarrow G L(k)$ are equivalent if they differ by an inner automorphism of $G L(k)$. We consider $G L(k) \subset G L(k+1) \subset \cdots \subset G L(k+r)$ in the usual way and thus consider a homomorphism $\rho: G \rightarrow G L(k)$ as, a fortiori, a homomorphism into $G L(k+r)$. We say that $\rho: G \rightarrow G L(k)$ and $\rho^{\prime}: G \rightarrow G L(r)$ are stably equivalent if they are equivalent considered as homomorphisms of $G$ into $G L(s), s$ sufficiently large. It follows by the reducibility theorem that if $\rho$ and $\rho^{\prime}$ are stably equivalent then both factor through $G L(\min (k, r))$ and are equivalent in $G L(\min (k, r))$.

Let $M$ be a $C^{\infty}$ manifold along with a continuous homomorphism $\gamma: G \rightarrow \operatorname{Diff}(M)$, the latter having the $C^{\infty}$ topology. Let $F(M)$ denote the set of points of $M$ fixed by every element of $G$ under the action of $G$ on $M$ induced by $\gamma$. Then $M$ (or more precisely the pair $(M, \gamma))$ will be called a $(G, \rho)$ manifold if for each $x \in F(M)$ the induced representation of $G$ on the tangent space $T M_{x}$ of $M$ at $x$ is stably equivalent to $\rho$. (This makes sense since the group of nonsingular linear transformations of $T M_{x}$ can be identified uniquely up to inner automorphisms with $G L($ dimension $(M))$.) Obviously this property involves only the stable equivalence class of $\rho$. If $F(M)=\varnothing$ then the condition is vacuously satisfied.

Let $M$ be a $(G, \rho)$ manifold of dimension $n$, let $T M$ denote the tangent bundle of $M$ and set $F(T M)=T M \mid F(M)$. Then the action of $G$ on $F(T M)$ via the differential induces (see $[10, \S 38.2]$ ) a redution of the structural group of the bundle $F(T M)$ from $G L(n)$ to $C(G)$, where $C(G)$ is the centralizer of $\rho(G)$ in $G L(n)$. A $(G, \rho)$ orientation of $M$ is a further reduction of the group of the bundle $F(T M)$ from $C(G)$ to $C(G)_{0}$, the component of the identity of $C(G)$. Note that if $F(M)$ is simply connected such a reduction always exists. In general, the obstruction to such a reduction lies in $H^{1}\left(F(M) ; Z_{2} \oplus Z_{2} \oplus \cdots \oplus Z_{2}\right)$ where the number of factors of $Z_{2}=$ $\boldsymbol{Z} / 2 \boldsymbol{Z}$ depends on $\rho$. Then number of distinct reductions is $a^{b}$ where $a$ = number of components of $C(G)$ and $b=$ number of components of $F(M)$.

An oriented $(G, \rho)$ isomorphism from one $(G, \rho)$ manifold $M$ to another $M^{\prime}$ is a $G$ equivariant diffeomorphism $f: M \rightarrow M^{\prime}$ which preserves the $(G, \rho)$ orientation.

A $(G, \rho)$ manifold $M$ is semi-free if $G$ acts freely on $M-F(M)$. Let $V_{\rho}^{n, k}$ denote the set of $(G, \rho)$ isomorphism classes of $(G, \rho)$ oriented semi-free $(G, \rho)$ manifolds $M$, where dimension of $M$ is $n$. Let $\mathscr{D}_{\rho}^{n, k}=\left\{M \in V_{\rho}^{n, k} \mid M\right.$ and $F(M)$, with $C^{\infty}$ triangulations, are combinatorially equivalent to disks $\}$ and $\mathscr{S}_{\rho}^{n, k}=\left\{M \in V_{\rho}^{n, k} \mid M\right.$ and $F(M)$, with $C^{\infty}$ triangulations, are combinatorially equivalent to spheres\}. 
The main aim of this paper is to obtain some results on the structure of the sets $\mathscr{D}_{\rho}^{n, k}$ and $\mathscr{S}_{\rho}^{n, k}$.

REMARK. We will often without warning use the same symbol $(M, \Delta, \Sigma$, etc.) to denote a $(G, \rho)$ oriented manifold, its underlying smooth manifold, its underlying action, and/or its $(G, \rho)$ isomorphism class in $\mathscr{D}_{\rho}^{n, k}$ or $\mathscr{S}_{\rho}^{n, k}$. We hope the context will make the intended meaning clear.

2. The groups $D S_{\rho}^{n, k}$ and $C \mathscr{S}_{\rho}^{n, \dot{k}}$ and additive structures on $\mathscr{S}_{\rho}^{n, k}$ and $\mathscr{D}_{\rho}^{n, k}$. The representation $\rho$ is equivalent to an orthogonal one, and we may as well assume $\rho=i \circ \rho_{0}$, where $\rho_{0}: G \rightarrow O(k)$ and $i$ is the natural inclusion of $O(k)$ into $G L(k)$. Thus $G$ acts on $S^{k-1}$ and $D^{k}$ via $\rho_{0}(G)$ and on $S^{n}$ and $D^{n+1}$ for $n \geqq k-1$ via the inclusion of $O(k)$ into $O(n+1)$. So $S^{n}$ and $D^{n+1}$ with this action are $(G, \rho)$ manifolds. They will be denoted by $S_{\rho}^{n, k}$ and $D_{\rho}^{n+1, k}$.

If $M \in V_{\rho}^{n k}$ (thus $M$ is semi-free) and if the dimension of $F(M)$ is $\mathrm{q}$ then $n-q$ is the smallest integer $t$ such that $\rho: G \rightarrow G L(k)$ factors through a representation $\rho^{\prime}: G \rightarrow G L(t)$. To simplify notation we assume $t=k$ (otherwise replace $\rho$ by $\rho^{\prime}$ ). If $V_{\rho}^{n, k}$ is nonempty for some $n$ then the action $\rho$ of $G$ on $S^{k-1}$ must be free and to avoid vacuity we now assume this is the case. (For $G \neq Z_{2}$ this forces $k$ to be even.) Then $S_{\rho}^{n, k}$ for $n \geqq k-1$ has fixed-point set $S^{n-k}$ (where $S^{-1}=\varnothing$ ), and $T S^{n} \mid S^{n-k}$ has a natural reduction to the trivial group. Thus $S_{\rho}^{n, k}$ represents an element in $V_{\rho}^{n, k}$ and thus in $\mathscr{S}_{\rho}^{n, k}$. Similarly, $D_{\rho}^{n+1, k}$ has $D^{n+1-k}$ as fixed-point set and represents an element of $\mathscr{D}_{\rho}^{n+1, k}$. Furthermore, the restriction of the oriented $(G, \rho)$ structure on $D_{\rho}^{n+1, k}$ to its boundary is just the $(G, \rho)$ oriented manifold $S_{\rho}^{n, k}$.

Let $D S_{\rho}^{n, k}$ for $n \geqq k-1$ be the set of equivalence classes of oriented $(G, \rho)$ isomorphisms $f$ of $S_{\rho}^{n, k}$ to itself, where two of them, $f$ and $f^{\prime}$, are equivalent if $f^{\prime} \circ f^{-1}: S^{n} \rightarrow S^{n}$ extends to an oriented $(G, \rho)$ isomorphism of $D_{\rho}^{n+1, k}$ to itself. Under composition of isomorphisms $D S_{\rho,}^{n,}$ is a group with the identity map as the unit.

It is possible, if one is careful, to define an addition on the sets $\mathscr{S}_{\rho}^{n k}$ and $\mathscr{D}_{\rho}^{n+1, k}$ for $n>k$, using equivalent connected sum and boundary connected sum of manifolds, respectively.

To do this we utilize the notion of $(G, \rho)$ orientation. First consider the $(G, \rho)$ oriented manifold $D_{\rho}^{n, k}$, with $n>k$. Now $D^{n} \subset R^{n}=R^{k} \times R^{n-k}$ and $h \in G$ acts on the point $(x, y) \in D^{n} \subset R^{k} \times R^{n-k}$ by $h(x, y)=(\rho(h) x, y)$. Let $j^{\prime}: D^{n-k} \rightarrow D^{n-k}$ be an orientation-reversing isometry, that is, $j^{\prime} \in O(n-k)-S O(n-k)$. Then $j: D^{n} \rightarrow D^{n}$ defined by $j(x, y)=\left(x, j^{\prime}(y)\right.$ ) (that is, $j=1 \times j^{\prime} \in 1 \times O(n-k) \subset O(k) \times$ $O(n-k) \subset O(n))$ is a $G$ equivariant diffeomorphism. The pullback of 
the standard $(G, \rho)$ orientation of $D_{\rho}^{n, k}$ by $j$ induces a new $(G, \rho)$ orientation, and $D_{\rho}^{n, k}$ with this new $(G, \rho)$ orientation will be denoted by $\hat{D}_{p}^{n, k}$. Note that if $G=\{e\}$ then this reduces to reversing the orientation in the usual sense, and in any case is independent of the particular choice of $j^{\prime}$.

Now let $M$ be any semi-free closed $(G, \rho)$ oriented manifold of dimension $n$. Let $x \in F(M)$. Then by [4] there exists a $G$ equivariant embedding $f: D_{\rho}^{n, k} \rightarrow M$ with $f(0)=x$. By replacing $f$ by $f \circ L$ for some $L \in C(G)-C(G)_{0}$, if necessary, we may assume that $f$ is $(G, \rho)$ orientation-preserving. The $G$ equivariant embedding $f \circ j: D_{\rho}^{n, k} \rightarrow M$ defines uniquely a new reduction of the structural group of $T M \mid F(M)_{x}$ to $C(G)_{0}$, where $F(M)_{x}$ is the connected component of $F(M)$ containing $x$. Thus picking one point out of each component of $F(M)$ determines a new $(G, \rho)$ orientation of $M . M$ with this new $(G, \rho)$ orientation will be denoted by $\hat{M}$. Note that $\hat{M}$ is defined only if dimension of $M>k$. (See [29] for $\operatorname{dim} M=k$.)

With these preliminaries out of the way we can now define connected sum and boundary connected sum in our category. Let $M$ and $M^{\prime}$ be two $(G, \rho)$ oriented manifolds of dimension $n$, with $n>k$. Let $f$ and $f^{\prime}$ be $G$ equivariant $(G, \rho)$ orientation-preserving embeddings $f: D_{\rho}^{n, k} \rightarrow M-\partial M$ and $f^{\prime}: D_{\rho}^{n, k} \rightarrow M^{\prime}-\partial M^{\prime}$. Set

$$
M+M^{\prime}=\left(M-f\left(\operatorname{Int} D^{n}\right)\right) \bigcup_{f^{\prime} j f-1 \mid f\left(\hat{o} D^{n}\right)}\left(M^{\prime}-f^{\prime}\left(\operatorname{Int} D^{n}\right)\right),
$$

where $j: D^{n} \rightarrow D^{n}$ is the orientation reversal defined above. After smoothing corners in the usual way, $M+M^{\prime}$ is a $(G, \rho)$ oriented manifold.

Now let $N$ and $N^{\prime}$ be two $(G, \rho)$ oriented manifolds of dimension $n+1$, with $n>k$. Let $f$ and $f^{\prime}$ be $G$ equivariant $(G, \rho)$ orientation-preserving embeddings $f: D_{\rho}^{n, k} \rightarrow \partial N$ and $f^{\prime}: D_{\rho}^{n, k} \rightarrow \partial N^{\prime}$. Set $N \oplus N^{\prime}=N U_{f^{\prime} j f^{-1}} N^{\prime}$, where the union is taken along the images of $f$ and $f^{\prime}$. Again after smoothing corners we get $N \oplus N^{\prime}$ as a $(G, \rho)$ oriented manifold.

The following are proved in $\S 5$.

Proposition 1. If $F(M)$ and $F\left(M^{\prime}\right)$ are connected, then the $(G, \rho)$ isomorphism class of $M+M^{\prime}$ is uniquely determined by $M$ and $M^{\prime}$, i.e., it is independent of the particular maps $f$ and $f^{\prime}$. Similarly if $F(N) \cap \partial N=F(\partial N)$ and $F\left(N^{\prime}\right) \cap \partial N^{\prime}=F\left(\partial N^{\prime}\right)$ are connected, the $(G, \rho)$ isomorphism class of $N \oplus N^{\prime}$ is independent of $f$ and $f^{\prime}$.

COROLlaRY. If $n>k$, then + as defined above induces a binary operation on $\mathscr{S}_{\rho}^{n, k}$ which is independent of the choices of basepoints 
$x$ and $x^{\prime}$ and embeddings $f$ and $f^{\prime}$. Under this operation $\mathscr{S}_{\rho}^{n, k}$ is an associative, commutative monoid with $S_{\rho}^{n, k}$ as the identity. (See [29] for the case $n=k$.) The same thing is true for $\mathscr{D}_{\rho}^{n+1, k}$ with $D_{\rho}^{n+1, k}$ as the identity.

REMARK. The above corollary is true because we are working in the category of $(G, \rho)$ oriented manifolds. It is not possible to drop this notion and still get a well-defined addition. (However, see Remark 1 in $\S 4$.)

We now define another set $C \mathscr{S}_{\rho}^{n, k}$ as follows: Let $\Sigma$ and $\Sigma^{\prime}$ represent elements of $\mathscr{S}_{\rho}^{n, k}$. We say $\Sigma$ is $h$-cobordant to $\Sigma^{\prime}$ if there is a $(G, \rho)$ oriented semi-free $(G, \rho)$ manifold $W$ such that $W$ and $F(W)$, with $C^{\infty}$ triangulations, are combinatorially equivalent to $S^{n} \times[0,1]$ and $S^{n-k} \times[0,1]$, respectively, and $\partial W$ as a $(G, \rho)$ oriented manifold is $(G, \rho)$ isomorphic to the disjoint union of $\Sigma$ and $\hat{\Sigma}^{\prime}$. Then $h$-cobordism is an equivalence relation and the equivalence classes are the elements of $C \mathscr{S}_{\rho}^{n, l}$. It is simple to check that addition preserves $h$-cobordism and hence induces a monoid structure on $C \mathscr{S}_{\rho}^{n, k}$ for $n>k$. Furthermore, the natural map $\nu: \mathscr{S}_{\rho}^{n, k} \rightarrow$ $C \mathscr{S}_{\rho}^{n, k}$ is an epimorphism of monoids. The usual proof (see [13]) goes through to show that $\Sigma+\hat{\Sigma}$ bounds an element in $\mathscr{D}_{\rho}^{n+1, k}$. Hence $\Sigma+\hat{\Sigma}$ is $h$-cobordant to the unit $S_{\rho}^{n, k}$ in $C \mathscr{S}_{\rho}^{n, k}$. Thus $C \mathscr{S}_{\rho}^{n, k}$ contains additive inverses and is an abelian group for $n>k$.

3. Some morphisms and an exact sequence. We now wish to examine the relations among the sets $D \mathscr{S}_{\rho}^{n, k}, \mathscr{S}_{\rho}^{n, k}, \mathscr{D}_{\rho}^{n+1, k}$ and $C \mathscr{S}_{\rho}^{n, k}$, which are monoids for $n>k$. We already have the surjection $\nu: \mathscr{S}_{\rho}^{n, k} \rightarrow C \mathscr{S}_{\rho}^{n, k}$. We define some other functions.

$$
\alpha: D S_{\rho}^{n, k} \longrightarrow \mathscr{S}_{\rho}^{n+1, k} \text {. }
$$

Define $\alpha$ as follows. Let $f: S_{o}^{n, k} \rightarrow S_{\rho}^{n, k}$ be an oriented $(G, \rho)$ isomorphism. Set $\alpha(f)=D_{\rho}^{n+1, k} \bigcup_{f} \hat{D}_{\rho}^{n+1, k}$, where $\partial D_{\rho}^{n+1, k}=S_{\rho}^{n, k}$ is identified with $\partial\left(\hat{D}_{\rho}^{n+1, k}\right)=\hat{S}_{\rho}^{n, k}$ under $f$. Then $\alpha(f) \in \mathscr{S}_{\rho}{ }^{n+1, k}$. It is not difficult to see that $\alpha$ is well-defined. We prove in $\S 5$ that it is an injection for $n \geqq k-1$ and a monomorphism of monoids for $n \geqq k$.

$$
\beta: \mathscr{S}_{\rho}^{n, k} \longrightarrow \mathscr{D}_{\rho}^{n, k} \text {. }
$$

Let $\Sigma$ represent an element of $\mathscr{S}_{\rho}^{n, k}$ and let $x \in F(\Sigma)$. Choose an equivariant $(G, \rho)$ oriented embedding $f: D_{\rho}^{n, k} \rightarrow \Sigma$ with $f(0)=x$. Set $\beta(\Sigma)=\Sigma-f\left(\operatorname{Int} D^{n}\right)$. If $n>k$, then $\beta$ induces a well-defined map from $\mathscr{S}_{\rho}^{n, k}$ to $\mathscr{D}_{\rho}^{n, k}$ which is a morphism for $n>k+1$. This follows from the proof of Proposition 1 in $\S 5$.

$\partial: \mathscr{D}_{\rho}^{n+1, k} \longrightarrow \mathscr{S}_{\rho}^{n, k}$. 
The boundary map $\partial$ is clearly well-defined for $n \geqq k-1$ and a morphism for $n>k$.

Proposition 2. The following sequence is exact for $n \leqq k$.

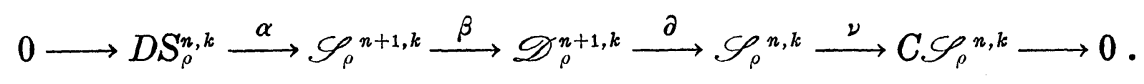

The proof is straightforward and will be omitted.

Note. $D S_{\rho}^{n, k}$ is a group for any $n$. For $n>k$ the first and last terms are groups while the other terms are monoids.

Complement. The above sequence (of sets) is also defined and exact for $n=k-1 \geqq 5$ and $G$ cyclic.

Proof. For $n=k-1$ and $\Sigma \in \mathscr{S}_{\rho}^{n+1, k}$ the fixed-point set $F(\Sigma)=S^{\circ}$ is just two points. The map $\beta$ is a priori not welldefined here, i.e., it may depend on the choice of $x \in S^{0}$. However, it follows easily from Milnor's application [21 Corollary 12.13] of the fixed-point formula of Atiyah-Bott $[1, \S 7]$ that for $n \geqq 5$ and $G$ cyclic, $\beta$ is well-defined and in fact is the constant map $\beta\left(\mathscr{S}_{\rho}{ }^{n+1, k}\right)=$ $D_{\rho}^{n+1, k} \in \mathscr{D}_{\rho}^{n+1, k}$. Then exactness of the sequence follows easily.

4. Main results. Our main result is a computation of $\mathscr{D}_{\rho}^{n, k}$ for $k \neq 2$ and $n$-dim $G \geqq 6$, where $\operatorname{dim} G=\operatorname{dimension}$ of $G$ as a manifold, and a partial computation for $n$-dim $G=5$ or $k=2$. The proof is in $\S 5$.

THeOREm A. Assume $k \neq 2$ and let $\pi=\pi_{0}(G)=G / G_{0}$ where $G_{0}$ is the component of the identity of $G$. Then there is a function

$$
\tau: \mathscr{D}_{\rho}^{n, k} \longrightarrow \mathrm{Wh}(\pi),
$$

where $\mathrm{Wh}(\pi)$ denotes the Whitehead group of $\pi$ (see [Milnor 4, $\S 6]$ ), such that

(1) $\tau$ is a morphism for $n>k+1$,

(2) if $n-\operatorname{dim} G \geqq 6$, then $\tau$ is a bijection,

(3) for $n$-dim $G=5$, the set $\mathscr{D}_{\rho}^{n, k}$ contains a subset $\mathscr{F}_{\rho}^{n, k}$ such that $\tau \mid \mathscr{F}_{\rho}^{n, k}$ is a bijection onto the subgroup $\tau\left(\mathscr{F}_{\rho}^{n, k}\right)=\{x+\bar{x} \mid x \in$ $\mathrm{Wh}(\pi)\}$, which has finite index in $\mathrm{Wh}(\pi)$ (see [21, §6.10 and §11.5]. For the definition of conjugation $\bar{x}$ see below and [21, p. 373 and p. 398].) If $n>k+1$ and $\mathrm{Wh}(\pi)$ is free abelian (e.g., if $\pi$ is cyclic, see [11] and [3, Prop. 4.14]), then $\mathscr{F}_{\rho}^{n, k}$ is a subgroup of the monoid $\mathscr{D}_{\rho}^{n, l}$ and $\tau \mid \mathscr{F}_{\rho}^{n, k}$ is an isomorphism onto its image. 
ADDENDUM. For $k=2$ and $n$-dim $G \geqq 6$, we have $\mathscr{E}_{p}^{n, 2}=\left\{D_{p}^{n, 2}\right\}$, where $\mathscr{E}_{\rho}^{n, 2}=\left\{\Delta \in \mathscr{D}_{\rho}^{n, 2} \mid \Delta-F(\Delta)\right.$ has the homotopy type of the circle and $\pi_{1}(\partial \Delta-\partial F(\Delta))$ is $\left.Z\right\}$.

REMARK 1. From the definition of $\tau$ (see $\S 5$ ) one sees that $\tau(\Delta)$ for $\Delta \in \mathscr{D}_{\rho}^{n, k}$ depends only on the action and not on its $(G, \rho)$ orientation. Thus, for $n$-dim $G \geqq 6$ and $k \neq 2$, the function from $\mathscr{D}_{\rho}^{n, k}$ to $\left|\mathscr{D}_{\rho}^{n, k}\right|$, defined by "forgetting" the $(G, \rho)$ orientation, is a bijection, where $\left|\mathscr{D}_{\rho}^{n, k}\right|$ denotes the set of equivariant diffeomorphism classes of semi-free actions of $G$ on $D^{n}$ with fixed-point set an $(n-k)$-disk and representation $\rho$ at the fixed points. This is because every such action can be $(G, \rho)$ oriented and $\tau$ is injective. In particular, any two $(G, \rho)$ orientations of such an action are related by an oriented $(G, \rho)$ isomorphism.

CoROLlary 1. A semi-free action of a compact Lie group $G$ on the $n$-disk, $n \geqq 6+\operatorname{dim} G$, with fixed-point set an $(n-k)$-disk, $k \neq 2$, is smoothly equivalent to a linear action if and only if its Whitehead torsion vanishes. Two such actions are smoothly equivalent if and only if they have equivalent linear representations at the fixed points and the same Whitehead torsion.

Proof. This follows immediately from Theorem A and Remark 1.

Corollary 2. If $\mathrm{Wh}(\pi)=\mathrm{Wh}\left(\pi_{0}(G)\right)=0$, then $\mathscr{D}_{\rho}^{n, k}=0$ and $D S_{\rho}^{n-1, k}=\mathscr{S}_{\rho}^{n, k}=C \mathscr{S}_{\rho}^{n, k}$ for $n \geqq 6+\operatorname{dim} G$ and $k \neq 2$. In particular, this is true if $\operatorname{dim} G>0$ or if $G=\boldsymbol{Z}_{2}, \boldsymbol{Z}_{3}, \boldsymbol{Z}_{4}$ or $\boldsymbol{Z}_{6}$.

Proof. For $n>k$ the first statement follows immediately from Theorem A and Proposition 2. In case $n=k$, we have $D S_{\rho}^{k-1, k}=$ $\mathscr{S}_{\rho}^{k, k}$. This gives $\mathscr{S}_{\rho}^{k, k}$ a group structure which is preserved by $\nu: \mathscr{S}_{\rho}^{k, k} \rightarrow C \mathscr{S}_{\rho}^{k, k}$. Since ker $\nu=0$, the result follows.

The second statement is true because if $\operatorname{dim} G>0$ then (see [5, p. 153]) $G=S^{1}, S^{3}$, or the normalizer of $S^{1}$ in $S^{3}$, so that, in any case, $\pi_{0}(G)=0, \boldsymbol{Z}_{2}, \boldsymbol{Z}_{3}, \boldsymbol{Z}_{4}$ or $\boldsymbol{Z}_{6}$. and thus $\mathrm{Wh}\left(\pi_{0}(G)\right)=0$ (see [11] and [3, Prop. 4.14]).

REMARK 2. The case $\operatorname{dim} G>0$ is proved in [5, VI. 9.1] without assuming $k \neq 2$.

CoROLlary 3. If $n \geqq 6+\operatorname{dim} G, n>k+1$ and $k \neq 2$, then $\mathscr{D}_{\rho}^{n, k}$ under $\oplus$ is an abelian group.

Proof. The additive inverse of an element $\Delta \in \mathscr{D}_{\rho}^{n, k}$ is $-\Delta=$ $\tau^{-1}(-\tau(\Delta))$. 
Caveat. It is not $\hat{\Delta}(=\Delta$ with opposite $(G, \rho)$ orientation as defined in $\S 2)$. In fact, $\Delta=\hat{\Delta}$ in $\mathscr{D}_{\rho}^{n, k}$. See Remark 1 .

CoRollary 4. If $n \geqq 5+\operatorname{dim} G, n>k$ and $k \neq 2$, then $\mathscr{P}_{\rho}^{n, k}$ is an abelian group under+.

Proof. This follows from an easy algebraic argument applied to the exact sequence of Proposition 2, knowing that $\mathscr{D}_{\rho}^{p+1, k}$ and $C . \mathscr{S}_{\rho}^{n, k}$ are groups.

Under the hypotheses of Corollary 4, all the monoids in the exact sequence of Proposition 2 are groups and all the morphisms are homomorphisms.

Let $d: \mathscr{D}_{\rho}{ }^{n+1, k} \rightarrow \mathscr{D}_{\rho}^{n, k}$ denote the composition $d=\beta \circ \partial$, which is defined for $n>k$ (and for $n=k$ if $n \geqq 5$ and $G$ is cyclic). For $n>k+1$ the function $d$ is a morphism and $d^{2}=0$. Under the hypotheses of Theorem $\mathrm{A}(2)$ we can interpret $d: \mathrm{Wh}(\pi) \rightarrow \mathrm{W}(\pi)$. We would like to give an algebraic description of $d$.

The action of $G$ on $R^{k}-0$ via the representation $\rho$ induces a homomorphism $v: G \rightarrow \operatorname{Aut}\left(\widetilde{H}_{k-1}\left(R^{k}-0\right)\right)=\operatorname{Aut}(Z)=Z_{2}=\{-1,1\}$. Clearly $G_{0}$ is in the kernel of $v$. Hence $v$ induces a homomorphism $w: \pi \rightarrow Z_{2}$. Then $w$ induces an anti-automorphism -, with square the identity, on $Z[\pi]$, the integral group ring of $\pi$, characterized by $\bar{a}=w(a) a^{-1}$ for all $a \in \pi \subset Z[\pi]$. This anti-automorphism of $Z[\pi]$ induces an automorphism of $\mathrm{Wh}(\pi)$ still denoted by - with $\overline{\bar{x}}=x$, for all $x \in \mathrm{Wh}(\pi)$. In our situation $G$ acts freely on $R^{k}-0$ and from this it is easy to show that $w(\pi)=1$ unless $G=Z_{2}$ in which case $\mathrm{Wh}(G)=0$.

The following is proved in $\S 5$.

Theorem B. Set $g=\operatorname{dim} G$. If $n>k$ and $k \neq 2$, then

$$
\tau(d \Delta)=\tau(\Delta)-(-1)^{n-g} \overline{\tau(\Delta)}
$$

for $\Delta \in \mathscr{D}_{\rho}^{n+1, k}$. Hence if in addition $n-g \geqq 6$, then $d: \mathscr{D}_{\rho}{ }^{n+1, k} \rightarrow$ $\mathscr{D}_{\rho}^{n, k}$ can be identified with the homomorphism $d: \mathrm{Wh}(\pi) \rightarrow \mathrm{Wh}(\pi)$ defined by $d(x)=x-(-1)^{n-g} \bar{x}$. This makes sense for $n-g=5$ (via Theorem $\mathrm{A}(3)$ ) and is true if $\mathrm{Wh}(\pi)$ is free abelian, e.g., if $\pi$ is cyclic.

Corollary 5. Let $G=Z_{m}$ and let $\rho: G \rightarrow G L(k), k \neq 2$, be a representation such that $G$ acts freely on $S^{k-1}$, i.e., $S^{k-1} / \rho(G)$ is a lens space. Then for $n$ odd, $n \geqq 5$ and $n \geqq k$, the function $\beta: \mathscr{S}_{\rho}^{n+1, k} \rightarrow$ $\mathscr{D}_{\rho}^{n+1, k}$ is zero. Hence,

$$
0 \longrightarrow \mathrm{Wh}\left(\boldsymbol{Z}_{m}\right) \stackrel{\partial_{\circ} \tau^{-1}}{\longrightarrow} \mathscr{S}_{\rho}^{n, k} \stackrel{\nu}{\longrightarrow} C \mathscr{S}_{\rho}^{n, k} \longrightarrow 0
$$


is a short exact sequence and $\alpha: D S_{\rho}^{n, k} \rightarrow \mathscr{S}_{\rho}^{n+1, k}$ is an isomorphism.

Proof. Since $\mathrm{Wh}\left(\boldsymbol{Z}_{m}\right)$ is free abelian, conjugation is the identity (see [21, Corollary 6.10]). It follows, therefore, from Theorems A and $\mathrm{B}$ that if $n \geqq 5$ is odd and $n>k \neq 2$ then $d: \mathscr{D}_{\rho}{ }^{n+1, k} \rightarrow \mathscr{D}_{\rho}{ }^{n, k}$ is injective (in fact, $d$ is multiplication by 2 in a torsion-free group). Furthermore, from the exact sequence of Proposition 2 it follows that $\beta: \mathscr{S}_{\rho}^{n+1, k} \rightarrow \mathscr{D}_{\rho}{ }^{n+1, k}$ is zero since $d$ is injective and $d \circ \beta=0$. Hence, the exact sequence breaks into

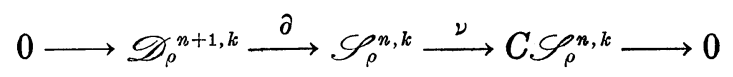

and

$$
0 \longrightarrow D S_{\rho}^{n}{ }^{k} \stackrel{\alpha}{\longrightarrow} \mathscr{S}_{\rho}^{n+1, k} \longrightarrow 0 \text {. }
$$

Applying Theorem A again completes the proof.

Corollary 6. Assume $n \geqq 5$ is odd, $k \geqq 4$ is even, $n-k \geqq-1$ and $m \geqq 5, m \neq 6$. Then there exist infinitely many distinct semifree actions of $\boldsymbol{Z}_{m}$ on $S^{n}$ with fixed-point set an $(n-k)$-sphere. In fact, the set of equivariant diffeomorphism classes of nonlinear semi-free actions of $\boldsymbol{Z}_{m}$ on $D^{n+1}$ with fixed-point set an $(n-k+1)$ disk is infinite and the restrictions of these actions to $\partial D^{n+1}=S^{n}$ are all distinct.

Proof. According to [11] $\mathrm{Wh}\left(\boldsymbol{Z}_{m}\right)$ has positive rank if $m \neq 1,2$, 3,4 , or 6 . Now, any smooth manifold $\Delta$ homeomorphic to $D^{n+1}$ is diffeomorphic to $D^{n+1}$ if $n \geqq 5$ by [32]. Thus for $n-k \geqq 1$ the result follows from Corollary 5 and Remark 1 . For $n=k-1$ we must show that $\partial \circ \tau^{-1}$ maps $\mathrm{Wh}\left(\boldsymbol{Z}_{m}\right)$ one-to-one into $\mathscr{S}_{\rho}^{k-1, k}$ (for $n>k$ this follows from exactness). To do this assume $\partial \Delta_{0}=\partial \Delta_{1}$ for $\Delta_{0}$, $\Delta_{1} \in \mathscr{D}_{\rho}^{k, k}$. Let $f: \partial \Delta_{0} \rightarrow \partial \Delta_{1}$ be an equivariant diffeomorphism. Glueing $\Delta_{0}$ and $\Delta_{1}$ together equivariantly along their boundaries produces an element $\Sigma \in \mathscr{S}_{\rho}^{k, k}$. It follows from the sum and duality theorems for Whitehead torsion (see [21, §7.4 and §10]) that $\tau(\beta(\Sigma))=$ $\tau\left(\Delta_{0}\right)-f_{*}\left(\overline{\tau\left(\Delta_{1}\right)}\right)$. But $f_{*}$ is the identity since $f$ is equivariant. Since conjugation is the identity in $\mathrm{Wh}\left(\boldsymbol{Z}_{m}\right)$ it follows from the Complement to Proposition 2 that $\tau\left(\Delta_{0}\right)-\tau\left(\Delta_{1}\right)=\tau(\beta(\Sigma))=0$. Hence $\Delta_{0}=\Delta_{1}$ as required. This completes the proof of Corollary 6 .

REMARK 3. It is easy to see that these free nonlinear actions of $Z_{m}$ on $S^{k-1}$ are the same as those constructed in [20], where Milnor showed that infinitely many of them are distinct, using Reidemeister torsion invariants, while we have observed that his later result 


\section{[21, Corollary 12.13] implies they are all distinct.}

From Theorem B it follows by a well-known result [8] that $\operatorname{ker} d: \mathscr{D}_{\rho}^{n, k} \rightarrow \mathscr{D}_{\rho}^{n-1, k}$ modulo im $d: \mathscr{D}_{\rho}^{n+1, k} \rightarrow \mathscr{D}_{\rho}^{n, k}$ is $H_{n-g}\left(\boldsymbol{Z}_{2} ; \mathrm{Wh}(\pi)\right)$ where $\boldsymbol{Z}_{2}$ acts on $\mathrm{Wh}(\pi)$ via conjugation -. The groups $H_{n}\left(\boldsymbol{Z}_{2} ; \mathrm{Wh}(\pi)\right)$ are of course periodic of period 2 .

Now $d \circ \beta=0$, so $\beta\left(\mathscr{S}_{\rho}^{n+1, k}\right) \subset \operatorname{ker} d$. Since $d=\beta \circ \partial$ we have a well-defined

$$
\psi_{1}: C \mathscr{S}_{\rho}^{n+1, k} \longrightarrow H_{n+1-g}\left(Z_{2} ; \mathrm{Wh}(\pi)\right) \text {. }
$$

Let $\psi_{2}$ be the composition

$$
D S_{\rho}^{n-1, k} \stackrel{\alpha}{\longrightarrow} \mathscr{S}_{\rho}^{n, k} \stackrel{\nu}{\longrightarrow} C \mathscr{S}_{\rho}^{n, k} .
$$

We have $\partial(\operatorname{ker} d) \subset \alpha\left(D S_{\rho}^{n-1, k}\right)$ and $\partial(\operatorname{im} d)=0$. Thus $\partial$ induces a $\operatorname{map} \psi_{3}: H_{n+1-g}\left(Z_{2} ; \mathrm{Wh}(\pi)\right) \rightarrow D S_{\rho}^{n-1, k}$.

Proposition 3. Assume $n-g=n-\operatorname{dim} G \geqq 6, n \geqq k+1$ and $k \neq 2$. Then the following sequence is exact

$$
\begin{aligned}
& \cdots \stackrel{\psi_{1}}{\longrightarrow} H_{n+r+2-g}\left(\boldsymbol{Z}_{2} ; \mathrm{Wh}(\pi)\right) \stackrel{\psi_{3}}{\longrightarrow} D S_{\rho}^{n+r, k} \\
& \quad \stackrel{\psi_{2}}{\longrightarrow} C \mathscr{S}_{\rho}^{n+r+1, k} \stackrel{\psi_{1}}{\longrightarrow} H_{n+r+1-g}\left(\boldsymbol{Z}_{2} ; \mathrm{Wh}(\pi)\right) \stackrel{\psi_{3}}{\longrightarrow} \cdots \\
& \quad \stackrel{\psi_{1}}{\longrightarrow} H_{n+1-g}\left(\boldsymbol{Z}_{2} ; \mathrm{Wh}(\pi)\right) \stackrel{\psi_{3}}{\longrightarrow} D S_{\rho}^{n-1, k} \stackrel{\psi_{2}}{\longrightarrow} C \mathscr{S}_{\rho}^{n, k} .
\end{aligned}
$$

Proof. This follows from Proposition 2 and Theorem B by an elementary algebraic argument.

5. Proofs. The proofs of this section depend on the following

LEMmA (Equivariant covering isotopy theorem). Let $N$ and $M$ be compact smooth manifolds with $\partial M=\varnothing$, and let $G$ be a compact Lie group acting smoothly on $N$ and $M$. Assume that $\lambda$ is an equivariant isotopy of $N$ in $M$, i.e., a smooth family of equivariant embeddings $\lambda_{t}: N \rightarrow M$. Then there exists an equivariant covering isotopy of $M$, i.e., an equivariant isotopy is of $M$ such that $\psi^{\prime}{ }^{\circ} \lambda_{0}=\lambda_{t}$ for $t \in[0,1]$.

Proof. By the (nonequivariant) covering isotopy theorem (see [22], [16] or [18]) there exists an isotopy $\varphi$ of $M$ such that $\varphi_{0}=$ identity and $\varphi_{t} \circ \lambda_{0}=\lambda_{t}$ for $t \in[0,1]$. Then by Theorem 3.1 of [5, Chap. VI] there exists an equivariant isotopy $\psi$ of $M$ such that $\psi_{t}\left|\lambda_{0}(N)=\varphi_{t}\right| \lambda_{0}(N)$. This completes the proof.

Proof of Proposition 1 and its corollary. We wish to show 
that the sum and the boundary sum operations for $(G, \rho)$ oriented manifolds are well-defined. We consider only the sum operation, the argument for boundary sum being the same except for trivial modifications.

Let $M$ and $M^{\prime}$ be two $n$-dimensional $(G, \rho)$ oriented manifolds. We wish to show that $M+M^{\prime}$ is defined uniquely up to oriented $(G, \rho)$ isomorphism and that + on isomorphism classes is commutative and associative, provided $F(M)$ and $F\left(M^{\prime}\right)$ are connected. What we have to show is that the result is independent of the $(G, \rho)$ equivariant orientation-preserving embeddings $f: D^{n} \rightarrow M$ and $f^{\prime}: D^{n} \rightarrow M^{\prime}$.

Suppose we replace $f$ by $\bar{f}: D^{n} \rightarrow M$, where $\bar{f}(0)=f(0)$ and $\bar{f}$ is a $(G, \rho)$ orientation-preserving embedding. Then uniqueness of equivariant tubular neighborhoods [5, p. 310] implies that $f$ is equivariantly isotopic to $f_{1}$, and $\bar{f}$ to $\bar{f}_{1}$, such that $f_{1}=\bar{f}_{1} \circ \psi$, where $\psi: D^{n} \rightarrow D^{n}$ is an equivariant linear map, i.e., $\psi \in C(G)$. Since $f$ and $\bar{f}$ were chosen as $(G, \rho)$ orientation-preserving, it follows that is $\in C_{0}(G)$. Thus $\psi$ is equivariantly isotopy to the identity. It follows that $f$ and $\bar{f}$ are equivariantly isotopic.

Now suppose we replace $f$ by $\bar{f}$ where $\bar{f}(0) \neq f(0)$. Since we assumed $F(M)$ is connected, there is a path in $F(M)$ joining $f(0)$ to $\bar{f}(0)$, that is, an equivariant isotopy of $\bar{f}(0)$ to $f(0)$. Using the equivariant covering isotopy theorem, we can then equivariantly isotop $\bar{f}$ to $\overline{\bar{f}}$ with $\overline{\bar{f}}(0)=f(0)$. Then the argument above shows $\overline{\bar{f}}$ is equivariantly isotopic to $f$. Hence, $\bar{f}$ is equivariantly isotopic to $f$ for any other $(G, \rho)$ equivariant orientation-preserving embedding $\bar{f}: D^{n} \rightarrow M$.

Thus we can apply the equivariant covering isotopy theorem to get an oriented $(G, \rho)$ isomorphism $h: M \rightarrow M$ with $h \circ f=\bar{f}$. This proves that the sum is well-defined up to $(G, \rho)$ oriented isomorphism class. Associativity and commutativity are immediate from the definition since we may assume $j=j^{-1}$.

Proof that $\alpha: D S_{\rho}^{n-1, k} \rightarrow \mathscr{S}_{\rho}^{n, k}$ is an injection for $n \geqq k$ and $a$ morphism for $n \geqq k+1$.

We assume $n \geqq k+1$ (a similar proof goes through for $n=k$ ). Recall that $\alpha$ is defined as follows. Given an oriented $(G, \rho)$ isomorphism $f: S_{\rho}^{n-1, k} \rightarrow S_{\rho}^{n-1, k}$, set $\alpha(f)=D_{0}^{n} \bigcup_{f} D_{1}^{n}$, where $D_{0}^{n}=D_{\rho}^{n, k}$ and $D_{1}^{n}=\hat{D}_{\rho}^{n, k}$. Now let $h: D_{0}^{n} \bigcup_{f} D_{1}^{n} \rightarrow D_{0}^{n} \bigcup_{f}, D_{1}^{n}$ be a $(G, \rho)$ oriented diffeomorphism. Since $n \geqq k+1$, the fixed-point set $F(\alpha(f))$ is connected. Hence by the proof of Proposition 1 we can equivariantly isotop $h$ to $h^{\prime}: D_{0}^{n} \mathrm{U}_{f} D_{1}^{n} \rightarrow D_{0}^{n} \mathrm{U}_{f}, D_{1}^{n}$ such that $h^{\prime} \mid D_{0}^{n}$ is inclusion. It follows that $h^{\prime \prime}=h^{\prime} \mid D_{1}^{n}: D_{1}^{n} \rightarrow D_{1}^{n}$ is a $(G, \rho)$ oriented isomorphism such that $h^{\prime \prime} \mid \partial D_{1}^{n}$ is exactly $f^{\prime} f^{-1}$. Hence $f^{\prime} f^{-1}$ extends 
over $D_{\rho}^{n, k}$ and thus $f=f^{\prime}$ in $D S_{\rho}^{n-1, k}$. Hence $\alpha$ is injective.

To show that $\alpha$ is a morphism of monoids one simply chooses carefully the disks used in constructing the equivariant connected sum of $(G, \rho)$ manifolds. For $\alpha(f)=D_{0}^{n} \bigcup_{f} D_{1}^{n}$ use the disk $\hat{D}_{1}^{n}$ while for $\alpha\left(f^{\prime}\right)=D_{0}^{n} \bigcup_{f^{\prime}} D_{1}^{n}$ use $D_{0}^{n}$. With this choice the construction for $\alpha(f)+\alpha\left(f^{\prime}\right)$ yields $\alpha\left(f^{\prime} \circ f\right)$ on the nose.

\section{Proof of Theorem A.}

DEFINITION OF $\tau$. Let $\Delta$ represent an element of $\mathscr{D}_{\rho}^{n, k}$. Let $\varepsilon: T \rightarrow \Delta$ be a closed $G$ equivariant tubular neighborhood of $F(\Delta)$ in $\Delta$. (For a semi-free action $F(\Delta) \neq \phi$ since no nontrivial group can act freely on a disk.) Let $\delta(T)$ be the associated sphere bundle to T. For $k \neq 2$, it follows easily that $\varepsilon(\delta(T)) \subset \Delta-\operatorname{Int}(\varepsilon(T))$ is a homotopy equivalence, while for $k=2$ the hypothesis of the Addendum to Theorem A implies this. Let $V=\varepsilon(\delta(T)) / G=\delta(T) / G=$ $D^{n-k} \times S^{k-1} / G$ and $W=(\Delta-\operatorname{Int}(\varepsilon(T))) / G$ be the decomposition manifolds. Since they are the base spaces of principal $G$ bundles the inclusion $c: V \rightarrow W$ is a homotopy equivalence, under our hypothesis. Hence (see [21, §9]) there is defined a Whitehead torsion invariant $\tau(\iota) \in \mathrm{Wh}\left(\pi_{1}(V)\right)$. By abuse of notion we define $\tau(\Delta)=\tau(\iota)$.

For $k>2$ we have that $\pi_{1}(V)=\pi_{1}\left(D^{n-k} \times S^{k-1} / G\right)$ is isomorphic to $\pi=\pi_{0}(G)=G / G_{0}=$ the group of covering transformations of the universal cover $\widetilde{V}=D^{n-k} \times S^{k-1} / G_{0}$. This isomorphism is unique up to inner automorphisms, which induce the identity automorphism of the Whitehead group. Hence there is a canonical identification $\mathrm{Wh}\left(\pi_{1}(V)\right)=\mathrm{Wh}(\pi)$ and we have $\tau(\Delta) \in \mathrm{Wh}(\pi)$.

From the uniqueness theorem for closed equivariant tubular neighborhoods, the equivariant covering isotopy theorem, the invariance of Whitehead torsion under diffeomorphism, and the fact that the canonical identification $\mathrm{Wh}\left(\pi_{1}(V)\right)=\mathrm{Wh}(\pi)$ is preserved under equivariant diffeomorphism, it follows that $\tau: \mathscr{D}_{\rho}^{n, k} \rightarrow \mathrm{Wh}(\pi)$ is well-defined for $k>2$.

If $k=2$, then $\pi_{1}(V)=Z$ or 0 , hence $\mathrm{Wh}\left(\pi_{1}(V)\right)=0$ by [11]. If $k=1$, then $G=Z_{2}$ so $\pi_{1}(V)=0$ and thus $\mathrm{Wh}\left(\pi_{0}(G)\right)=0$. Therefore $\tau$ is well-defined in all cases.

Proof that $\tau$ is a morphism for $n>k+1$. We have defined $\tau: \mathscr{D}_{\rho}^{n, k} \rightarrow \mathrm{Wh}(\pi)$. For $n>k+1$ we have a monoid structure on both the domain and range of $\tau$. We wish to show that $\tau$ is a morphism. To do this, let $\Delta$ and $\Delta^{\prime}$ represent elements of $\mathscr{D}_{\rho}^{n, k}$. We can assume that $\Delta, \Delta^{\prime}$ and $D_{\rho}^{n-1, k} \times[0,1]$ (with $G$ acting trivially on $[0,1])$ are pairwise disjoint. Let $f: D_{\rho}^{n-1, k} \rightarrow \partial \Delta$ and $f^{\prime}: D_{\rho}^{n-1, k} \rightarrow \partial \Delta^{\prime}$ be equivariant $(G, \rho)$ oriented embeddings. Set $\Delta_{0}=\left(D_{\rho}^{n-1, k} \times[0,1 / 2]\right)$ 
$\mathrm{U}_{f} \Delta$, where $f$ glues $D_{\rho}^{n-1, k} \times[0,1 / 2]$ to $\Delta$ along $D_{\rho}^{n-1, k} \times\{0\}=f\left(D_{\rho}^{n-1, k}\right)$, and $\Delta_{0}^{\prime}=\left(D_{\rho}^{n-1, k} \times[1 / 2,1]\right) \bigcup_{f^{\prime}} \Delta^{\prime}$, where $f^{\prime}$ glues $D_{\rho}^{n-1, k} \times[1 / 2,1]$ to $\Delta^{\prime}$ along $D_{\rho}^{n-1, k} \times\{1\}=f^{\prime}\left(D_{\rho}^{n-1, k}\right)$. Then clearly as elements of $\mathscr{D}_{\rho}^{n, k}$ we have $\Delta_{0}=\Delta$ and $\Delta_{0}^{\prime}=\Delta^{\prime}$. Furthermore, $\Delta \oplus \Delta^{\prime}$ is represented as an element of $\mathscr{D}_{\rho}^{n, k}$ by $\Delta_{0} \cup \Delta_{0}^{\prime}$, where $\Delta_{0}$ is glued to $\Delta_{0}^{\prime}$ along $D_{\rho}^{n-1, k} \times\{1 / 2\}=$ $\Delta_{0} \cap \Delta_{0}^{\prime}$.

Now choose equivariant tubular neighborhoods $T_{1}$ of $F\left(\Delta_{0}\right)$ and $T_{2}$ of $F\left(\Delta_{0}^{\prime}\right)$ such that $T=T_{1} \cup T_{2}$ and $T_{3}=T_{1} \cap T_{2}$ are equivariant tubular neighborhoods of $F\left(\Delta_{0} \cup \Delta_{0}^{\prime}\right)$ and $F\left(D_{\rho}^{n-1, k} \times\{1 / 2\}\right)$, respectively. Using the notation of the definition of $\tau$, we have $W=W_{1} \cup W_{2}$, $W_{3}=W_{1} \cap W_{2}, V=V_{1} \cup V_{2}$, and $V_{3}=V_{1} \cap V_{2}$. Now by the sum theorem of [14] $\tau(\iota)=\tau\left(c_{1}\right)+\tau\left(\iota_{2}\right)-\tau\left(\iota_{3}\right)$, where $\iota: V \rightarrow W$ and $\iota_{i}: V_{i} \rightarrow W_{i}$ are inclusions, $i=1,2,3$. But $\tau\left(\iota_{3}\right)=0$ since $V_{3}=$ $D^{n-k-1} \times S^{k-1} / G$ and $W_{3}=V_{3} \times[0,1]$, with $\iota_{3}(x)=(x, 0)$ for $x \in V_{3}$. Hence

$$
\begin{aligned}
\tau\left(\Delta \oplus \Delta^{\prime}\right) & =\tau\left(\Delta_{0} \oplus \Delta_{0}^{\prime}\right)=\tau(\iota)=\tau\left(\iota_{1}\right)+\tau\left(\iota_{2}\right)=\tau\left(\Delta_{0}\right)+\tau\left(\Delta_{0}^{\prime}\right) \\
& =\tau(\Delta)+\tau\left(\Delta^{\prime}\right) .
\end{aligned}
$$

This completes the proof.

Proof that $\tau$ is surjective for $n \geqq 6+\operatorname{dim} G$. $G$ acts freely on $S^{k-1}$ under $\rho$ and hence freely on $S^{k-1} \times D^{n-k}$ via $h(x, y)=(\rho(h) x, y)$, where $h \in G$. This action extends to an action on $D^{k} \times D^{n-k}$ defined by the same formula.

Set $V=\left(S^{k-1} \times D^{n-k}\right) / G=S^{k-1} / G \times D^{n-k}$. If $n$-dim $G=1+$ dimension of $V \geqq 6$ then Sallings' construction [37] (see [21, § 11.1]) gives a manifold $W$ which is an $h$-cobordism between $V$ and $V^{\prime}$, with $V \cap V^{\prime}=\phi$ and $\partial W=V \cup V^{\prime} \cup V^{\prime \prime}$, where $V^{\prime \prime}$ is a product cobordism between $\partial V$ and $\partial V^{\prime}$, such that the Whitehead torsion $\tau(W, V)$ is equal to any preassigned element $\tau_{0}$ of $\mathrm{Wh}(\pi)=\mathrm{Wh}\left(\pi_{1}(V)\right)$. Let $p: \widetilde{W} \rightarrow W$ be the principal $G$ bundle over $W$ induced from the $G$ bundle $S^{k-1} \times D^{n-k} \rightarrow V$ by a deformation retraction of $W$ onto $V$. Then $p^{-1}(V)=S^{k-1} \times D^{n-k}$ with the given action of $G$ on it. Let $i$ denote the identity map of $p^{-1}(V)$. Then after smoothing corners $D^{k} \times D^{n-k} \bigcup_{i} \widetilde{W}$ becomes a contractible manifold with 1-connected boundary and hence by [32] is diffeomorphic to $D^{n}$. The actions of $G$ on the summands piece together to give the required action $\Delta$ of $G$ on $D^{n}$, with $\tau(\Delta)=\tau_{0}$, proving surjectivity (compare [20]).

Proof of part (3): the case $n$-dim $G=5$. For $n$-dim $G=5$, Stallings' construction is replaced by the "wedge" construction of Siebenmann-Sondow [31] (extended easily to manifolds with boundary) which provides invertible $h$-cobordisms between 4 -manifolds with any torsion of the form $x+\bar{x}$. The rest of the above recipe for $n$-dim 
$G \geqq 6$ goes through unchanged, proving that there is a function $\sigma: H \rightarrow \mathscr{D}_{\rho}^{n, k}$ such that $\tau \circ \sigma=$ identity, where $H$ is the subgroup $H=\{x+\bar{x} \mid x \in \mathrm{Wh}(\pi)\}$ of $\mathrm{Wh}(\pi)$. Set $\mathscr{F}_{\rho}^{n, k}=\sigma(H)$.

If $\mathrm{Wh}(\pi)$ has no elements of finite order, then for every $h \in H$ there exists a unique $x \in \mathrm{Wh}(\pi)$ such that $h=x+\bar{x}$. (Proof: If $x+\bar{x}=x_{0}+\bar{x}_{0}$, then $2\left(x-x_{0}\right)=(x-\bar{x})-\left(x_{0}-\bar{x}_{0}\right)$. But $x-\bar{x}$ and $x_{0}-\bar{x}_{0}$ have finite order by [21, Corollary 6.10]. Therefore, so does $x-x_{0}$, so $x=x_{0}$ ) Then the uniqueness theorem for $h$-cobordisms (see [21, §11.3]) implies that $\sigma$ is a morphism from the group $H$ to the monoid $\mathscr{D}_{\rho}^{n, k}$ if $n>k+1$ (compare the proof of invertibility in [31]). Hence $\mathscr{F}_{\rho}{ }^{n, k}=\sigma(H)$ is a group and $\tau \mid \mathscr{F}_{\rho}^{n, k}=\sigma^{-1}: \mathscr{F}_{\rho}^{n, k} \rightarrow H$ is an isomorphism. This completes the proof.

Proof that $\tau$ is injective for $n$-dim $G \geqq 6$. Using the notation of the definition of $\tau$, let $\varepsilon^{\prime \prime}: T^{\prime \prime} \rightarrow \partial \Delta$ be a closed $G$ equivariant tubular neighborhood of $\partial F(\Delta) \subset \partial \Delta$ such that $T \mid \partial F(\Delta)=T^{\prime} \subset \operatorname{Int} T^{\prime \prime}$ and $\varepsilon^{\prime \prime}\left|T^{\prime}=\varepsilon\right| T^{\prime}$. Introducing a corner along $\delta\left(T^{\prime \prime}\right) / G$ we have that $W$ is an $h$-cobordism between $V$ and $V^{\prime}=\left(\partial \Delta-\operatorname{Int} \varepsilon^{\prime \prime}\left(T^{\prime \prime}\right)\right) / G$, with $\partial W=W \cup V^{\prime} \cup V^{\prime \prime}$, where $V^{\prime \prime}$ is a product cobordism between $\partial V$ and $\partial V^{\prime}$.

Now given $\Delta_{1}, \Delta_{2} \in \mathscr{D}_{\rho}^{n, k}$ we apply this construction to each and get $h$-cobordisms $\left(W_{i} ; V_{i}, V_{i}^{\prime}\right), i=1,2$. Each $T_{i}$ is a $G$ equivariant bundle over a disk $D^{n-k}$, so $T_{i}$ when considered as a bundle with group $C(G)$ is still trivial, i.e., each $T_{i}=D^{k} \times D^{n-k}$ with $G$ action defined by $h(x, y)=(\rho(h) x, y)$ for $h \in G$. It follows that, up to isotopy, there is a well-defined equivariant diffeomorphism $f: T_{1} \rightarrow T_{2}$ which we may assume is $(G, \rho)$ orientation-preserving (since otherwise we may replace $f$ by $L \circ f$ for some $\left.L \in C(G)-C(G)_{0}\right)$. Then $f \mid \delta\left(T_{1}\right): \delta\left(T_{1}\right) \rightarrow \delta\left(T_{2}\right)$ covers a diffeomorphism $f_{0}: \delta\left(T_{1}\right) / G \rightarrow \delta\left(T_{2}\right) / G$.

If $\tau\left(A_{1}\right)=\tau\left(\Delta_{2}\right)$ then, by definition, the inclusions $\iota_{1}: V_{1} \rightarrow W_{1}$ and $\iota_{2}: V_{2} \rightarrow W_{2}$ have the same torsion invariant. Since $n$-dim $G=\operatorname{dimension}$ of $W_{i} \geqq 6$, the uniqueness theorem for $h$-cobordisms implies that $f_{0}: V_{1} \rightarrow V_{2}$ extends to a diffeomorphism $f_{1}: W_{1} \rightarrow W_{2}$. Then by elementary bundle theory this lifts to a $G$ equivariant diffeomorphism of principal bundles $\tilde{f}_{1}: \widetilde{W}_{1} \rightarrow \widetilde{W}_{2}$ such that $\tilde{f}_{1}\left|\widetilde{V}_{1}=f\right| \delta\left(T_{1}\right)$. Thus $f$ and $\widetilde{f}_{1}$ piece together to yield an oriented $(G, \rho)$ isomorphism $f \cup \widetilde{f}_{1}: \Delta_{1} \rightarrow \Delta_{2}$. Hence $\tau$ is one-to-one for $n$-dim $G \geqq 6$. This completes the proof of Theorem A.

Proof of the Addendum. It follows easily from the definition of $\mathscr{E}_{\rho}{ }^{n, 2}$ that the above construction applied to any element $\Delta \in \mathscr{E}_{\rho}{ }^{n, 2}$ yields an $h$-cobordism $\left(W ; V, V^{\prime}\right)$. In defining $\tau$ we saw that $\mathrm{Wh}\left(\pi_{1}(V)\right)$ is trivial. Hence the $s$-cobordism theorem of [2], [17] and [37] (see [12]) implies that $W$ is a product cobordism. The 
above proof then shows that $\Delta$ is the trivial element $\Delta=D_{\rho}^{n, 2} \in \mathscr{E}_{\rho}^{n, 2}$. This completes the proof.

Proof of Theorem B. We wish to calculate the Whitehead torsion $\tau(d \Delta)$ for $\Delta \in \mathscr{D}_{\rho}^{n+1, k}$ and $d: \mathscr{D}_{\rho}^{n+1, k} \rightarrow \mathscr{D}_{\rho}^{n, k}$. Let $f: D_{\rho}^{n, k} \rightarrow \partial \Delta$ be an equivariant $(G, \rho)$ oriented embedding. Then $d \Delta=\partial \Delta-f\left(\operatorname{Int} D_{\rho}^{n, k}\right) \epsilon$ $\mathscr{D}_{\rho}^{n, k}$. Choose a closed equivariant tubular neighborhood $\varepsilon: T \rightarrow \Delta$ of the fixed-point set $F(\Delta)$ in $\Delta$ such that $T \mid F\left(f\left(D_{\rho}^{n, k}\right)\right)$ and $T \mid F(d \Delta)$ are closed equivariant tubular neighborhoods of $F\left(f\left(D_{o}^{n, k}\right)\right)$ and $F(d \Delta)$ in $f\left(D_{\rho}^{n, k}\right)$ and $d \Delta$, respectively. Set $W=(\Delta-\varepsilon(\stackrel{\circ}{T})) / G, W_{1}=$ $\left(f\left(D_{\rho}^{n, k}\right)-\varepsilon\left(\stackrel{T}{T} \mid F\left(f\left(D_{\rho}^{n, k}\right)\right)\right)\right) / G$ and $W_{2}=(d \Delta-\varepsilon(\dot{T} \mid F(d \Delta))) / G$ where $\stackrel{\circ}{T}$ is the open disk bundle associated to $T$, i.e., $\stackrel{T}{T}=T-\delta(T)$. Also set $V=\delta(T) / G, V_{1}=\left(\delta(T) \mid F\left(f\left(D_{\rho}^{n, k}\right)\right)\right) / G$ and $V_{2}=(\delta(T) \mid F(d \Delta)) / G$. Let

$$
\begin{aligned}
& \iota_{2}: V_{2} \longrightarrow W_{2} \\
& \iota: V \longrightarrow W \\
& i_{1}: W_{2} \longrightarrow W \\
& i_{2}: W_{1} \cup W_{2} \longrightarrow W \\
& i_{3}: V_{2} \longrightarrow V \\
& i_{4}: V_{1} \longrightarrow W
\end{aligned}
$$

denote the inclusions. We wish to compare $\tau(d \Delta)=\tau\left(\iota_{2}\right)$ with $\tau(\Delta)=\tau(\iota)$. Now $\partial W=V \cup W_{1} \cup W_{2}$, with $V \cap\left(W_{1} \cup W_{2}\right)=\partial V=$ $\partial\left(W_{1} \cup W_{2}\right)=V_{1} \cup V_{2}$. Since $W_{1}=V_{1} \times[0,1]$, where $V_{1}$ is identified with $V_{1} \times 0$ and $W_{1} \cap W_{2}=V_{1} \times 1$, it follows that $\tau\left(i_{1}\right)=\tau\left(i_{2}\right)$. By the duality formula of $[21, \S 10]$ we have $\tau(\iota)=(-1)^{n-g+1} \overline{\tau\left(i_{2}\right)}$, since $n-g+1=\operatorname{dim} W$. Hence $\tau(\iota)=(-1)^{n-g+1} \overline{\tau\left(i_{1}\right)}$. Now factor the inclusion $V_{2} \rightarrow W$ in two ways:

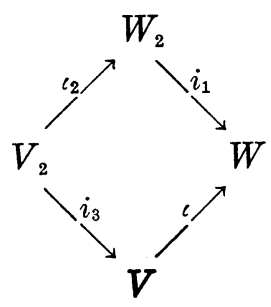

Hence (see 21, §7]) $\tau\left(\iota_{2}\right)+\tau\left(i_{1}\right)=\tau\left(i_{3}\right)+\tau(\iota)$. On the other hand we can also apply duality to $i_{3}$ to get $\tau\left(i_{3}\right)=(-1)^{\mathrm{dim} V_{1}} \overline{\tau\left(i_{4}\right)}$. However, $V=D^{n+1-k} \times S^{k-1} / G$ and $V_{1}=D^{n-k} \times S^{k-1} / G$ and $i_{4}$ is the natural inclusion. Hence $\tau\left(i_{4}\right)=0$. Therefore $\tau\left(i_{3}\right)=0$. Thus $\tau\left(c_{2}\right)+\tau\left(i_{1}\right)=\tau(\iota)$. Therefore $\tau(d \Delta)=\tau\left(i_{2}\right)=\tau(\iota)+(-1)^{n-g+1} \overline{\tau(\iota)}=\tau(\Delta)+(-1)^{n-g} \overline{\tau(\Delta)}$, as required.

For $n-g=5$ the "wedge" construction of [31] is used in the 
proof of Theorem A (3) to produce an action $\sigma(h) \in \mathscr{F}_{\rho}^{n, k} \subset \mathscr{D}_{\rho}^{n, k}$ such that $\tau(\sigma(h))=h$, for any $h \in H=\{x-\bar{x} \mid x \in \mathrm{Wh}(\pi)\} \subset \mathrm{Wh}(\pi)$. It is not difficult to see that $\sigma(h)=d \tau^{-1}(x)$ for some $x \in \mathrm{Wh}(\pi)$ such that $h=x+\bar{x}$. In other words, given $x \in \mathrm{Wh}(\pi)$ the wedge construction yields the same element $\sigma(x+\bar{x})=d \tau^{-1}(x)=\beta\left(\partial\left(\tau^{-1}(x)\right)\right) \in \mathscr{D}_{\rho}^{n, k}$ as does our construction of taking the action $\tau^{-1}(x) \in \mathscr{D}_{\rho}^{n+1, k}$ which has Whitehead torsion equal to $x$, then restricting the action to the boundary $\partial\left(\tau^{-1}(x)\right)$ and finally removing (via $\beta$ ) a copy of the trivial action $D_{\rho}^{n, k}$ at a fixed point. Thus we have

$$
\sigma(H)=\mathscr{F}_{\rho}^{n, k} \subset d\left(\mathscr{D}_{\rho}^{n+1, k}\right) \subset \tau^{-1}(H)
$$

because $\tau(d \Delta)=\tau(\Delta)-(-1)^{n-g} \overline{\tau(\Delta)} \in H$ since $n-g=5$ is odd.

Now if $\mathrm{Wh}(\pi)$ is free abelian $\sigma^{-1}=\tau \mid \mathscr{F}_{\rho}^{n, k}: \mathscr{F}_{\rho}^{n, k} \rightarrow H$ is an isomorphism by Theorem A (3). Moreover, $\tau \circ d \circ \tau^{-1}: \mathrm{Wh}(\pi) \rightarrow H$ is an isomorphism since it is $x \mapsto 2 x$ in a free abelian group. Therefore, $d\left(\mathscr{D}_{\rho}^{n+1, k}\right)=\mathscr{F}_{\rho}^{n, k}$ and $d: \mathscr{D}_{\rho}^{n+1, k} \rightarrow \mathscr{F}_{\rho}^{n, k}$ is the isomorphism $d=$ $\sigma \circ\left(\tau \circ d \circ \tau^{-1}\right) \circ \tau$. This completes the proof of Theorem B.

\section{REFERENCES}

1. M. F. Atiyah and R. Bott, A Lefschetz fixed point formula for elliptic complexes, II. Applications, Ann. of Math., 88 (1968), 451-491.

2. D. Barden, The structure of manifolds, Thesis, Cambridge University, 1963.

3. H. Bass, J. Milnor and J.-P. Serre, Solution of the congruence subgroup problem for $\mathrm{SL}_{n}(n \geqq 3)$ and $\mathrm{SP}_{2 n}(n \geqq 2)$, Publ. Inst. des Hautes Etudes Sci., \#33 (1967).

4. S. Bochner, Compact groups of differentiable transformations, Ann. of Math., 46 (1945), 372-381.

5. G. E. Bredon, Introduction to compact transformation groups, Academic Press, New York, 1971.

6. W. Browder, Differential topology of higher dimensional manifolds, Amer. Math. Soc., Colloquium Lectures, 1977.

7. W. Browder and T. Petrie, Diffeomorphisms of manifolds and semifree actions on homotopy spheres, Bull. Amer. Math. Soc., 77 (1971), 160-163.

8. H. Cartan and S. Eilenberg, Homological Algebra, Princeton Univ. Press, Princeton, N. J., 1956.

9. E. H. Connell, D. Montgomery and C. T. Yang, Compact groups in $E^{n}$, Ann. of Math., 80 (1964), 94-103; Correction, Ann. of Math., 81 (1965), 194.

10. P. E. Conner and E. E. Floyd, Differentiable Periodic Maps, Springer-Verlag, Berlin and New York, 1964.

11. G. Higman, The units of group rings, Proc. London Math. Soc., 46 (1940), 231-248.

12. M. Kervaire, Le théorème de Barden-Mazur-Stallings, Comment. Math. Helv., 40 (1965), 31-42.

13. M. Kervaire and J. Milnor, Groups of homotopy spheres, Ann. of Math., 77 (1963), 504-537.

14. K. W. Kwun and R. H. Szczarba, Product and sum theorems for Whitehead torsion, Ann. of Math., 82 (1965), 183-190.

15. R. Lashof and M. Rothenberg, G-smoothing theory, Proc. Amer. Math. Soc., Summer Inst., Stanford, 1976, to appear.

16. E. Lima, On the local triviality of the restriction map for embeddings, Comment. 
Math. Helv., 38 (1963-64), 163-164.

17. B. Mazur, Relative neighborhoods and the theorems of Smale, Ann. of Math., 77 (1963), 232-249.

18. J. Milnor, Differentiable structures, Lecture Notes, Princeton Univ., 1961.

19. , Two complexes which are homeomorphic but combinatorially distinct, Ann. of Math., 74 (1961), 575-590.

20. - Some free actions of cyclic groups on spheres, Differential Analysis, Tata Institute and Oxford Univ. Press, 1964, 37-42.

21. — Whitehead torsion, Bull. Amer. Math. Soc., 72 (1966), 358-426.

22. R. Palais, Local triviality of the restriction map for embeddings, Comment. Math. Helv., 34 (1960), 305-312.

23. M. Rothenberg, Actions des groups de Lie sur les variétés différèntiables, Lecture Notes, Université de Paris-Orsay, 1969-70.

24. — Differentiable group actions on spheres, Proc. Adv. Study Inst. Alg. Top., Aarhus, (1970), 455-475.

25. - Torsion invariants and finite transformation groups, Proc. Amer. Math. Soc., Summer Inst., Stanford, 1976, to appear.

26. R. Schultz, Semi-free circle actions and the degree of symmetry of homotopy spheres, Amer. J. Math., 93 (1971), 829-839.

27. — Homotopy sphere pairs admitting semi-free differentiable actions, Amer. J. Math., 96 (1974), 308-323.

28. - Differentiable group actions on homotopy spheres, Invent. Math., 31 (1975), 105-128.

29. M. Sebastiani, Sur les actions à deux points fixes de groupes finis sur les spheres, Comment Math. Helv., 45 (1970), 405-439.

30. J. Shaneson, Product formulas for $L_{n}(\pi)$, Bull. Amer, Math. Soc., 76 (1970), 787-791.

31. L. Siebemann and J. Sondow, Some homeomorphic sphere pairs that are combinatorially distinct, Comment. Math. Helv., 41 (1966-67), 261-272.

32. S. Smale, On the structure of manifolds, Amer. J. Math., 84 (1962), 387-399.

33. J. Sondow, Disproof of the Hauptvermutung for manifold pairs, Thesis, Princeton University, 1965. Abstract p. 6753 v. 26 no. 11, Order no. 66-05014, Xerox University Microfilms, 300N. Zeeb Rd., Ann Arbor, Mich. 48106.

34. - Actions of cyclic groups on spheres, Notices Amer. Math. Soc., 12 (1965), 618.

35. - Actions of cyclic groups on spheres, Proc. I. C. M., (1966), §8, 13.

36. Simple homotopy types of n-knots, to appear.

37. J. Stallings, On infinite processes leading to differentiability in the complement of a point, Differential and Combinatorial Topology ( $A$ Symposium in honor of $M$. Morse), Princeton Univ. Press, Princeton, N.J., 1965, 245-255.

38. T. Stewart, Fixed point sets and equivalence of differantiable transformation groups, Comment. Math. Helv., 38 (1963), 6-13.

39. V. Vijums, Characteristic subspheres of differentiable group actions on homotopy spheres, Ph.D. Thesis, Rutgers University, 1977.

40. J. Wolf, Spaces of constant curvature, 2nd ed., pub. by the author, Univ. of Calif., Berkeley, Calif., 1972.

Received February 10, 1978.

UNIVERSIUY OF CHICAGo

Chicago, IL 60637

AND

Columbia UNIVERSity

New YoRK, NY 10027 


\section{PACIFIC JOURNAL OF MATHEMATICS}

\section{EDITORS}

DONALD BABBITT (Managing Editor)

University of California

Los Angeles, CA 90024

HUGO RossI

University of Utah

Salt Lake City, UT 84112

C. C. Moore and ANDrew OGG

University of California

Berkeley, CA 94720
J. DUGUNDJI

Department of Mathematics University of Southern California Los Angeles, CA 90007

R. FINN and J. MILGRAM

Stanford University

Stanford, CA 94305

\section{ASSOCIATE EDITORS}
E. F. BeCKENBACH
B. H. NeumanN
F. WOLF
K. YOSHIDA

\section{SUPPORTING INSTITUTIONS}

UNIVERSITY OF BRITISH COLUMBIA CALIFORNIA INSTITUTE OF TECHNOLOGY UNIVERSITY OF CALIFORNIA MONTANA STATE UNIVERSITY UNIVERSITY OF NEVADA, RENO NEW MEXICO STATE UNIVERSITY OREGON STATE UNIVERSITY UNIVERSITY OF OREGON

\author{
UNIVERSITY OF SOUTHERN CALIFORNIA \\ STANFORD UNIVERSITY \\ UNIVERSITY OF HAWAII \\ UNIVERSITY OF TOKYO \\ UNIVERSITY OF UTAH \\ WASHINGTON STATE UNIVERSITY \\ UNIVERSITY OF · WASHINGTON
}

The Supporting Institutions listed above contribute to the cost of publication of this Journal, but they are not owners or publishers and have no responsibility for its content or policies.

Mathematical papers intended for publication in the Pacific Journal of Mathematics should be in typed form or offset-reproduced, (not dittoed), double spaced with large margins. Please do not use built up fractions in the text of the manuscript. However, you may use them in the displayed equations. Underline Greek letters in red, German in green, and script in blue. The first paragraph or two must be capable of being used separately as a synopsis of the entire paper. Please propose a heading for the odd numbered pages of less than 35 characters. Manuscripts, in triplicate, may be sent to any one of the editors. Please classify according to the scheme of Math. Reviews, Index to Vol. 39. Supply name and address of author to whom proofs should be sent. All other communications should be addressed to the managing editor, or Elaine Barth, University of California, Los Angeles, California, 90024.

50 reprints to each author are provided free for each article, only if page charges have been substantially paid. Additional copies may be obtained at cost in multiples of 50 .

The Pacific Journal of Mathematics is issued monthly as of January 1966. Regular subscription rate: $\$ 84.00$ a year (6 Vols., 12 issues). Special rate: $\$ 42.00$ a year to individual members of supporting institutions.

Subscriptions, orders for numbers issued in the last three calendar years, and changes of address should be sent to Pacific Journal of Mathematics, P.O. Box 969, Carmel Valley, CA 93924, U.S.A. Older back numbers obtainable from Kraus Periodicals Co., Route 100, Millwood, NY 10546.

PUBLISHED BY PACIFIC JOURNAL OF MATHEMATICS, A NON-PROFIT CORPORATION

Printed at Kokusai Bunken Insatsusha (International Academic Printing Co., Ltd.). 8-8, 3-chome, Takadanobaba, Shinjuku-ku, Tokyo 160, Japan.

Copyright (C) 1979 by Pacific Journal of Mathematics Manufactured and first issued in Japan 


\section{Pacific Journal of Mathematics}

\section{Vol. 84, No. $2 \quad$ June, 1979}

Somesh Chandra Bagchi and Alladi Sitaram, Spherical mean periodic

functions on semisimple Lie groups ........................ 241

Billy Joe Ball, Quasicompactifications and shape theory............. 251

Maureen A. Bardwell, The o-primitive components of a regular ordered permutation group ................................ 261

Peter W. Bates and James R. Ward, Periodic solutions of higher order

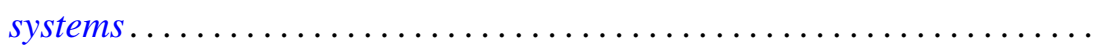

Jeroen Bruijning, A characterization of dimension of topological spaces by totally bounded pseudometrics......................... 283

Thomas Farmer, On the reduction of certain degenerate principal series

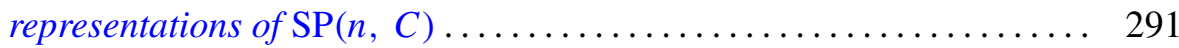

Richard P. Jerrard and Mark D. Meyerson, Homotopy with m-functions . . . . 305

James Edgar Keesling and Sibe Mardesic, A shape fibration with fibers of

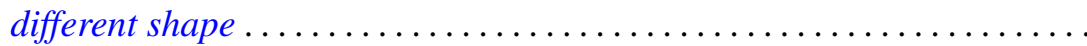

Guy Loupias, Cohomology over Banach crossed products. Application to bounded derivations and crossed homomorphisms ...............

Rainer Löwen, Symmetric planes ........................ 367

Alan L. T. Paterson, Amenable groups for which every topological left

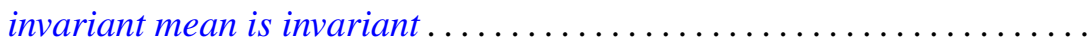

Jack Ray Porter and R. Grant Woods, Ultra-Hausdorff H-closed extensions

Calvin R. Putnam, Operators satisfying a $G_{1}$ condition .

Melvin Gordon Rothenberg and Jonathan David Sondow, Nonlinear smooth representations of compact Lie groups..............

Werner Rupp, Riesz-presentation of additive and $\sigma$-additive set-valued measures.

A. M. Russell, A commutative Banach algebra of functions of generalized variation

Judith D. Sally, Superregular sequences

Patrick Shanahan, On the signature of Grassmannians . . 